\title{
Light coupling in polymer nanofibers: from single-photon emission to random lasing
}

\begin{abstract}
A. Camposeo, M. Gaio, M. Moffa, M. Montinaro, M. Castro-Lopez, et al.
\end{abstract}

A. Camposeo, M. Gaio, M. Moffa, M. Montinaro, M. Castro-Lopez, V. Fasano, R. Sapienza, D. Pisignano, "Light coupling in polymer nanofibers: from single-photon emission to random lasing," Proc. SPIE 10101, Organic Photonic Materials and Devices XIX, 101010E (16 February 2017); doi: $10.1117 / 12.2263662$

SPIE. Event: SPIE OPTO, 2017, San Francisco, California, United States 


\title{
LIGHT COUPLING IN POLYMER NANOFIBERS: FROM SINGLE-PHOTON EMISSION TO RANDOM LASING
}

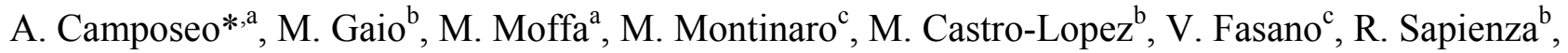 \\ D. Pisignano ${ }^{\mathrm{a}, \mathrm{c}}$ \\ ${ }^{a}$ NEST, Istituto Nanoscienze-CNR, Piazza San Silvestro 12, I-56127 Pisa (Italy); ${ }^{b}$ Department of \\ Physics, King's College London, Strand, London WC2R 2LS, United Kingdom; 'Dipartimento di \\ Matematica e Fisica "Ennio De Giorgi”, Università del Salento, via Arnesano, I-73100 Lecce (Italy)
}

\begin{abstract}
The understanding of the phenomena underlying the interaction of photons with dielectric, metallic and hybrid microand nano-structures and the development of advanced fabrication tools have paved the way to the realization of complex, nanostructured photonic structures, with tailored and exotic absorption and emission properties. Among such nanostructured materials, polymer nanofibers have intriguing and specific properties: they can embed molecular and quantum dot light sources, they can transport light among distant emitters and they can be arranged in 2-dimensional and 3-dimensional architectures in a controlled fashion, forming complex networks of interacting light emitters. However, coupling of light with polymer nanofibers depends on many variables, being often limited by the arrangement and positioning of the nanoscale light-sources, and by the fiber geometry. Here we report on the fabrication of active polymer nanofibers with improved surface properties and controlled geometry by electrospinning. Polarization and momentum spectroscopy of light emitted by molecular compounds and single quantum dots embedded in electrospun polymer fibers, evidence that efficient, nanostructured photon sources with targeted polarization and coupling efficiency can be realized in nanofiber-based photonic environments.
\end{abstract}

Keywords: electrospinning, nanofibers, single-photon sources, waveguides, lasers.

\section{INTRODUCTION}

Sub-wavelength dielectric structures are emerging as powerful building blocks for the fabrication of new optical materials [1]. These photonic structures are revolutionizing the management of light at micro- and nanoscale. For instance, individual dielectric nanostructures (nanowires, nanopillars, nanofibers, nanorings and nanodisks) or their assemblies in complex, often periodic, structures can enhance local optical fields [2], allow the permittivity and permeability of the resulting materials to be engineered [1] and, when coupled to light sources, can effectively direct the spontaneous emission of the coupled emitters [3]. Compared to metallic nanostructures which exploit plasmonic modes to guide and confine light in sub-diffraction volumes [4], dielectric nanostructures are not affected by energy dissipation due to light absorption, and in principle they can transport light even over macroscopic distances without significant losses, as required for high-performance nanophotonics. These properties motivate research on these nanostructures for controlling far-field spatial mode profiles, divergence and light extraction efficiency of both single-photon and laser sources [5,6]. In this framework, nanofibers and nanowires have the potential for combining long-range light propagation and local field enhancement, thus offering practical solutions for the control and management of light at sub-wavelength scales. In addition, nanofibers can be coupled to molecular and nanostructured emitters either by near-field light coupling or by directly embedding the light sources into the dielectric nanostructure, being the latter option generally more advantageous for achieving high coupling efficiencies [7,8]. Indeed, the main challenges in realizing effective interfaces with nanofibers are related to the integration of the light emitters (individual or in localized ensembles) in the dielectric structures. This has to be carried out by a gentle approach, possibly without using harsh chemical or high temperatures during processing in order to preserve the emission properties of the molecular or nanostructured light sources. Also, nanofibers and nanowires are to be tightly controlled in their structural uniformity, since variations of the fiber size along its length and surface roughness are strongly detrimental to light propagation.

*andrea.camposeo@nano.cnr.it; phone+39 0832298207; www.nanojets.eu 
In this respect, the use of tapered structures has allowed several intriguing effects primed by light sources coupled to optical nanofibers to be investigated [9]. A coupling efficiency up to $30 \%$ has been achieved by gently positioning quantum dot single-photon emitters on the surface of optical nanofibers, achieving values comparable to the maximum calculated ones for near-field evanescent coupling [10]. Optical nanofibers have been also coupled to laser-cooled atoms by evanescent fields to realize one-dimensional optical lattice traps [11]. More recently, breaking of mirror symmetry in light scattered by an individual Au nanoparticle deposited on the surface of an optical nanofiber was demonstrated by exploiting the strong transverse confinement of the light and spin-orbit coupling [12]. While featuring very low propagation losses (single mode transmission of $99.95 \%$ [13]), tapered optical nanofibers are mainly limited by the nearfield coupling to the molecular or nanostructured light emitters and by the difficulty of building complex 2-dimensional (2D) and 3-dimensional (3D) networks of them. Polymer nanofibers mechanically drawn from solution offer additional degrees of freedoms, because they can be molded or assembled in complex shapes or networks, and light emitters can be incorporated by simply adding the active components in the starting solution. To date, wavelength-converted waveguides [14], optical sensors [15] and lasers [16] have been realized by solution-drawn polymer nanofibers as building blocks. In addition, polymer nanofibers can be produced by electrostatic spinning, a method which allows a polymer filament to be drawn from a solution by means of electrostatic fields [17,18]. In this approach, a polymer jet is extruded through a metallic spinneret, biased by a high voltage $(\sim$ tens of $\mathrm{kV})$, and it is stretched by the applied electric field to generate solid polymer fibers with diameters ranging from few tens of $\mathrm{nm}$ to micrometers. Electrospun polymer fibers can be produced in continuous runs and can be collected on suitable metallic plates or patterned substrates as individual fibers or as $2 \mathrm{D}$ and 3D networks of ordered or randomly-oriented filaments [19]. Moreover, ordered arrays of light-emitting polymer nanofibers with controlled geometries and fiber positioning can be produced by so-called near-field electrospinning [20]. Similarly to solution-drawing, light emitters such as organic molecules and inorganic colloidal nanoparticles can be embedded in electrospun fibers following addition in solution. While many light sources have been reported by doping electrospun nanofibers with quantum dots, nanowires, organic dyes, and conjugated polymers [21], little has been done to engineering light coupling and consequently control emission.

Here, we focus on single-photon and laser sources based on electrospun polymer nanofibers. Individual polymer fibers doped with single quantum dots are fabricated with improved light coupling efficiency [22] and effective transport of single photons over distances in the range 10-100 microns. The complex dynamics of the polymer jets formed during the electrospinning process, which include a stretching of the entangled polymer network with a concomitant increase of the polymer density at the jet center [23], is exploited to direct most of the dopant quantum dots along the fiber axis, thus improving the coupling efficiency. Laser dyes are also embedded in polymer nanofibers and their emission amplified by stimulated emission. Random assemblies of the dye-doped polymer nanofibers in 3D nonwoven mats feature multimode random laser emission.

\section{METHODS}

Single-photon light sources were produced by electrospinning from a solution of poly(methyl methacrylate) (PMMA, Sigma Aldrich) doped with CdSeTe colloidal quantum dots (Invitrogen QD800). The polymer is used as transparent matrix, whereas the nanoparticles provide emission peaked at $790 \mathrm{~nm}$. PMMA is dissolved in chloroform, and mixed with a $1 \mathrm{nM}$ solution of emissive quantum dots and $30 \mathrm{mg}$ of the tetrabutylammonium iodide (TBAI, Sigma Aldrich) organic salt [24], used to obtain sub-micron fibers with uniform diameters. Fibers were produced by delivering the solution at constant rate $\left(0.5 \mathrm{~mL} \mathrm{~h}^{-1}\right)$ through a $27 \mathrm{G}$ gauge stainless steel needle, by using a syringe pump (Harvard Apparatus, Holliston, MA). The spinneret was biased at $8 \mathrm{kV}$ with respect to a $\mathrm{Cu}$ collector. The spinneret-collector distance was $10 \mathrm{~cm}$. Free-standing fibers were suspended through a transmission electron microscopy (TEM) grid (TAAB Laboratories Equipment Ltd) mounted on glass coverslip substrates. Electrospinning experiments were performed in laboratory conditions, with ambient temperature $\left(25^{\circ} \mathrm{C}\right)$ and a relative humidity about $40 \%$.

Dye-doped nanofibers were composed by a polyacrylonitrile (PAN, Sigma Aldrich) matrix doped with Rhodamine 6G (R6G, Exciton), providing light emission and optical gain. Specifically, PAN with $0.5 \%(\mathrm{w} / \mathrm{w})$ R6G was dissolved in dimethylformamide (Sigma-Aldrich, $10 \% \mathrm{w}: \mathrm{v}$ concentration) and spun through a $21 \mathrm{G}$ stainless steel needle. A $16 \mathrm{kV}$

bias was applied between the needle and the collector, and the solution flow rate was kept at $10 \mu \mathrm{L} \mathrm{min}{ }^{-1}$. Fiber mats, with thicknesses ranging from tens to hundreds of microns, were deposited on quartz substrates $\left(1 \times 1 \mathrm{~cm}^{2}\right)$.

The morphology of fibers was investigated by scanning electron microscopy (SEM) and atomic force microscopy (AFM). SEM measurements were performed by a FEI Nova NanoSEM 450 system, equipped with a scanning transmission electron microscopy (STEM) detector. AFM characterization was carried out in tapping-mode by using a 
Nanoscope IIIa controller with a Multi Mode head (Veeco), and cantilevers with tips of $8 \mathrm{~nm}$ nominal curvature and a resonant frequency of about $200 \mathrm{kHz}$. Single-photon nanofiber sources were characterized by confocal microscopy, exciting isolated quantum dots with a pulsed laser emitting at $634 \mathrm{~nm}$ (pulse duration $=100 \mathrm{ps}$ and repetition rate $=2.5$ $\mathrm{MHz})$ through an air objective (NA $=0.95)$ or an oil immersion objective $(\mathrm{NA}=1.45)$, while the light emitted was collected by the same objective. Lasing spectra were measured by exciting the mats of nanofibers with the third harmonic of a pulsed Nd:YAG laser, emitting at $355 \mathrm{~nm}$ (pulse duration=10 ns and repetition rate $=10 \mathrm{~Hz}$ ), focused on the sample in a stripe shape. The emission was collected from the excited edge of the sample by a lens, coupled to an optical fiber and analyzed spectrally by a monochromator, equipped with a charge coupled device (CCD) detector. All lasing measurements were performed in vacuum ambient (pressure below $10^{-4} \mathrm{mbar}$ ) in order to avoid photo-oxidation processes which might alter emission properties.

\section{RESULTS AND DISCUSSION}

Electrospun PMMA fibers have diameters in the range of $300 \mathrm{~nm}-1 \mu \mathrm{m}$. The diameter is uniform along the length of individual fibers over distances of the order of millimeters (with variations of about $15 \%$ along a fiber length of $1 \mathrm{~mm}$ ). This is favored by the addition of the organic salt in the spun solutions, which increases the conductivity and allows small and uniform fibers to be obtained [25]. We carefully investigated the effect of the salt addition on the emission properties of the CdSeTe quantum dots, by measuring their photoluminescence spectra in PMMA thin films with and without the addition of the organic salt. Such analysis did not evidence significant spectral changes. Another issue regards the positioning of the quantum dots into the fibers. Figure 1 shows STEM micrographs of PMMA electrospun fibers doped with aggregates of CdSeTe colloidal quantum dots. Here, aggregates of CdSeTe nanoparticles (size in the range 10-30 nm) allowed STEM imaging to be improved for the developed hybrid system. These results evidence that nanoparticles are mainly positioned nearby the fiber central region, namely at the core. The arrangement of nanoparticles within the electrospun nanofibers is mediated by the polymer mass transport, which is in turn determined by the complex dynamics of the polymer solution jet. Indeed, both theoretical calculations and experimental analysis of the polymer jet during a typical electrospinning process [26, 27] show the occurrence of a substantial axial stretching of the fluid filament composed by entangled polymer chains, which induces a contraction of the polymer network towards the jet center and a concomitant increase of the local density. For flexible polymer chains the increase of density at the jet central regions occurs at distances of the order of a few $\mathrm{mm}$ from the needle [27], and it is retained in the ultimately deposited nanofibers [23]. Such behavior might favor the transport of nanoparticles towards the jet axis, as here observed. In addition, the surface of fibers probed by AFM is found to be smooth with a measured surface roughness (root mean square) of about $3 \mathrm{~nm}$, as shown in the inset of Figure 1b. Such low roughness values favor the transport of single-photons to distances in the range $10-100 \mu \mathrm{m}$ as discussed below.
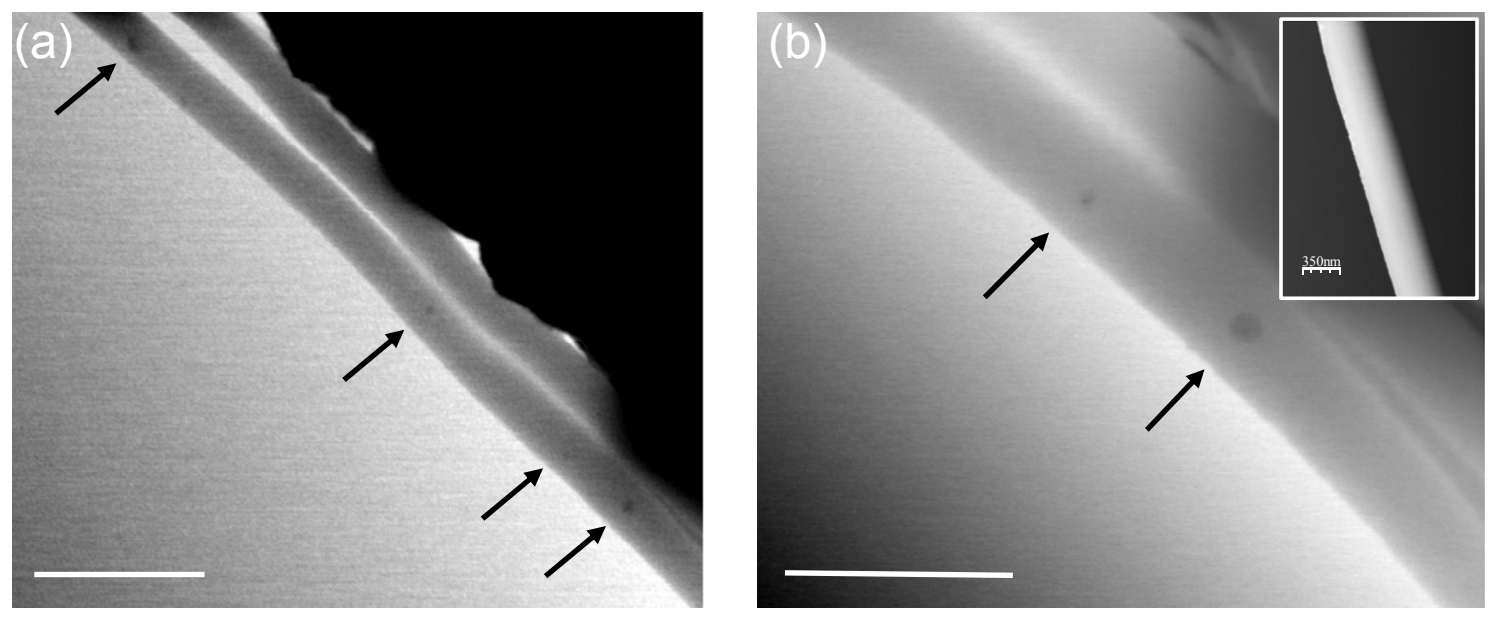

Figure 1. STEM micrographs of PMMA nanofibers, embedding quantum dots nano-aggregates and deposited on a TEM grid. The arrows highlight the positions of the embedded nanoparticles. Scale bars: (a) $1 \mu \mathrm{m}$, (b) $500 \mathrm{~nm}$. Inset: AFM topographic micrograph of an electrospun PMMA fiber doped with quantum dots. Scale bar: $350 \mathrm{~nm}$. 
When isolated quantum dots are embedded into PMMA electrospun fibers, single-photon emission is found with a typical saturation count rate of $42 \mathrm{kphotons} / \mathrm{s}$ at excitation intensity of $7 \mathrm{~kW} \cdot \mathrm{cm}^{-2}$ [22]. Direct transport of single-photons along the nanofiber can be investigated by imaging the laser-excited emission for a filament lying on glass substrate when purposely cut by a razor blade, as displayed in Figure 2. Here we show two emission micrographs, with the excitation (marked by the red circles) located at the quantum dot position (Figure 2a) and at the cleaved tip of the fiber (Figure 2b). In both cases, the widefield image clearly evidences the transport of light along the fiber as better discussed in the following. The corresponding intensity profiles along the fiber are shown in Figure $2 \mathrm{c}$ and $2 \mathrm{~d}$, respectively. In particular, Fig. $2 \mathrm{c}$ allows for quantifying the relative intensity of the light collected at the cleaved tip $\left(I_{t}\right)$, compared to the intensity collected directly at the quantum dot location $\left(I_{s}\right)$. The ratio $I_{t} / I_{s}=12 \%$ leads to an estimate of the coupling constant $\beta=24 \%$, assuming a similar collection efficiency for the two spots. The coupling constant indicates the fraction of the photons emitted by the quantum dots that is channeled into the fiber waveguide. The measured values are in good agreement with the values calculated for quantum dots embedded in polymer fibers, predicting values of $\beta$ up to $53 \%$ for a fiber with $400 \mathrm{~nm}$ diameter [22]. More quantitative measurements of $\beta$ were performed by momentum spectroscopy, which allows the coupling constants of individual nanofiber modes to be evaluated. To this aim we measured the angular intensity distribution of the light emitted by a single quantum dot in a nanofiber deposited on a glass substrate. These maps have intensity maxima at specific wave-vectors, related to the coupled modes as a consequence of the orthogonality of the nanofiber modes in $k$-space. Therefore, the intensity collected in momentum space at specific $k$ vectors provides a direct estimation of the photons channeled in a specific mode [22]. $\beta$ constants of individual modes can be calculated as the ratio between the mode-specific emission intensity and the total emission intensity. By such approach we obtained for the fundamental mode $\beta_{01}$ values up to $31 \%$. Moreover, the fluorescence measured at the tip of the fiber, $\sim 10 \mu \mathrm{m}$ away from the quantum dot (Fig.s 2a and 2c), highlights single-photon transport along the fiber length. In addition, Fig.s $2 \mathrm{~b}$ and $2 \mathrm{~d}$ demonstrate the possibility of long-range addressing of the emitter, exciting the quantum dot through light coupled at distant regions (fiber tip in Fig. 2b) and waveguided. For single-photon sources, polymer nanofibers are advantageous, because of their broadband response, their capability of efficiently channeling the light emitted by the quantum dot in a bandwidth of more than $\sim 100 \mathrm{~nm}$, and room temperature operation.

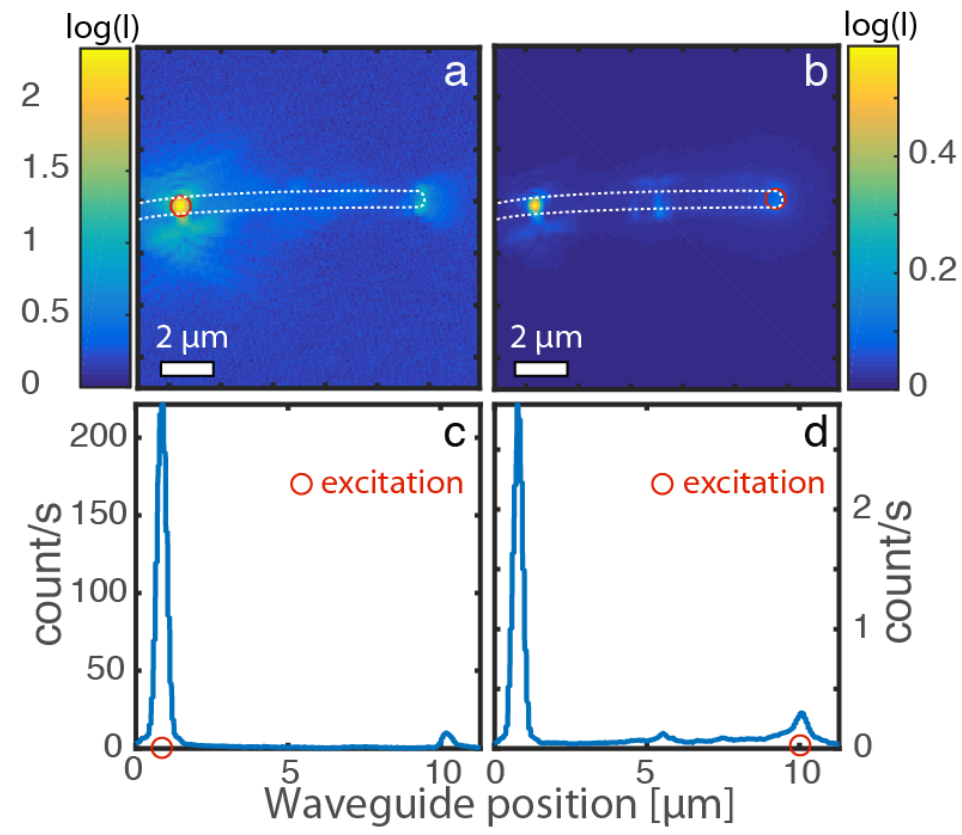

Figure 2. Coupling of the quantum dot to a nanofiber. (a, b): Wide-field fluorescence images of an intentionally cut nanofiber deposited on a glass substrate. In (a) the exciting laser is focused on a quantum dot embedded in the fiber, whereas in (b) the laser is moved to the fiber tip end, as marked by the red circles. (c, d): Corresponding intensity line profiles along the fibers in (a) and (b), respectively. When exciting directly the quantum dot (c), the bright spot at the fiber tip, which is $\sim 10 \mu \mathrm{m}$ away, indicates light transport with a relative intensity of $12 \%$. Instead, when illuminating the fiber tip (d), part of the excitation laser light is coupled into the fiber, it is guided toward the distant quantum dot and it is able to excite its emission. 
The composition and assembly of electrospun nanofibers can be varied and tailored to target specific photonic applications, not limited to single-photon sources. Recently, electrospun nanofibers featuring optical gain have been reported in a broad spectral range, for wavelengths up to the near infrared [28], by doping the fibers with suitable organic molecules. Optical gain was obtained in uniaxially aligned fibers, but such nanostructures can also be collected as mats of randomly oriented filaments, constituting a complex, 3D optical material with concomitant optical gain and disorder, where random lasing [29] effects can be investigated. We produced fibers of PAN with average size of $570 \mathrm{~nm}$ (standard deviation of the diameter distribution $=230 \mathrm{~nm}$ ), and emission peaked at about $570 \mathrm{~nm}$. Upon pulsed excitation with incident fluence of few $\mathrm{mJ} \mathrm{cm}{ }^{-2}$, we observed the rise of a band with full width at half maximum (FWHM) of $6 \mathrm{~nm}$, significantly smaller than the width of the spontaneous emission spectrum. The band is composed by different sharp peaks, with linewidth $<0.5 \mathrm{~nm}$, as for multimode random lasers, which are clearly distinguishable even after averaging over many single-shot lasing spectra (Figure 3a). The behavior of a sequence of single-shot emission spectra is shown in Figure $3 \mathrm{~b}$, highlighting that these peaks are almost stable in wavelength, with minor shot-to-shot intensity fluctuations. Lasing emission has been reported in electrospun filaments with different configurations, including randomly formed cavities [30], and attributed to different mechanisms which included the generation of Fabry-Pérot structures across individual filaments and the operation of ring resonators. Here, the 3D structure can provide even more complex and non-planar lasing architectures and inter-fiber coupling, extending further the possibility of obtaining random lasing in nanofibrous optical materials. Extensive analysis of intensity characteristics as well as of the spectral correlations in these devices are currently underway in our laboratories.

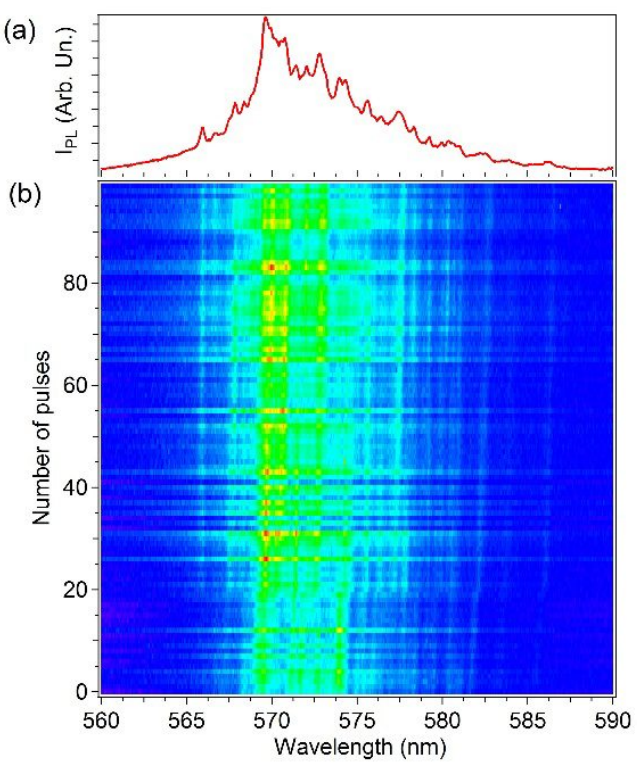

Figure 3. Lasing spectral features of electrospun nanofibers with optical gain. (a) Example of an emission spectrum collected from a mat of PAN/R6G nanofibers. This profile is obtained by averaging on 100 single-shot spectra. (b) Sequence of 100 single-shot emission spectra collected from a PAN/R6G nanofiber mat. Excitation fluence $=5 \mathrm{~mJ} / \mathrm{cm}^{2}$.

\section{CONCLUSIONS}

In summary, electrospun nanofibers are novel dielectric materials which provide a platform for realizing miniaturized light sources, ranging from single-photon sources to random lasers. Embedding nanoscale emitters, such as individual quantum dots, in electrospun nanofibers unveils coupling efficiencies of single-photons well above the values achievable by near-field evanescent coupling. In addition, the nanofibers can transport the coupled single-photons over distances of hundreds of micrometers, which is interesting in view of interconnecting many single quantum emitters in networks of polymer nanofibers designed for room temperature operation. These complex structures are also relevant for lasing applications, if suitable gain materials are embedded into the fibers. The lasing emission spectra of mats feature multimode emission, whose origin can be rationalized by an in-depth investigation of light transport, diffusion and localization in the 3D nanofibrous material. 
Acknowledgments. The research leading to these results has received funding from the Engineering and Physical Sciences Research Council (EPSRC), from the European Union's Seventh Framework Programme (FP7/2007-2013) EU Project People and from the European Research Council under the European Union's Seventh Framework Programme (FP/2007-2013)/ERC Grant Agreement n. 306357 (ERC Starting Grant "NANO-JETS"). The authors also acknowledge the Apulia Regional Projects 'Networks of Public Research Laboratories', WAFITECH (09) and M. I. T. T. (13). R. Manco is acknowledged for PAN/R6G sample preparation.

\section{REFERENCES}

[1] Jahani, S. and Jacob, Z., “All-dielectric metamaterials,” Nat. Nanotechnol. 11, $23-36$ (2016).

[2] Bakker, R. M., Permyakov, D., Yu, Y. F., Markovich, D., Paniagua-Domínguez, R., Gonzaga, L., Samusev, A., Kivshar, Y., Luk'yanchuk, B. and Kuznetsov, A. I., "Magnetic and electric hotspots with silicon nanodimers," Nano Lett. 15, 2137-2142 (2015).

[3] Moitra, P., Yang, Y., Anderson, Z., Kravchenko, I. I., Briggs, D. P. and Valentine, J., "Realization of an alldielectric zero-index optical metamaterial," Nat. Photon. 7, 791-795 (2013).

[4] Schuller, J. A., Barnard, E. S., Cai, W., Chul Jun, Y., White J. S. and Brongersma, M. L., "Plasmonics for extreme light concentration and manipulation," Nat. Mater. 9, 193 (2010).

[5] Babinec, T. M., Hausmann, B. J. M., Khan, M., Zhang, Y., Maze, J. R., Hemmer, P. R. and Lončar, M. A., "Diamond nanowire single-photon source," Nat. Nanotechnol. 5, 195-199 (2010).

[6] Eaton, S. W., Fu, A., Wong, A. B., Ning, C.-Z. and Yang, P., "Semiconductor nanowire lasers," Nat. Rev. Mater. 1, 16028 (2016).

[7] Yalla, R., Le Kien, F., Morinaga, M. and Hakuta, K., "Efficient channeling of fluorescence photons from single quantum dots into guided modes of optical nanofiber," Phys. Rev. Lett. 109, 063602 (2012).

[8] Faez, S., Türschmann, P., Haakh, H. R., Götzinger, S. and Sandoghdar, V., "Coherent interaction of light and single molecules in a dielectric nanoguide," Phys. Rev. Lett. 113, 213601 (2014).

[9] Nayak, K. P., Melentiev, P. N., Morinaga, M., Kien, F. L., Balykin, V. I. and Hakuta, K., "Optical nanofiber as an efficient tool for manipulating and probing atomic fluorescence," Opt. Express 15, 5431-5438 (2007).

[10] Klimov, V. V. and Ducloy, M., "Spontaneous emission rate of an excited atom placed near a nanofiber," Phys. Rev. A 69, 013812 (2004).

[11] Vetsch, E., Reitz, D., Sagué, G., Schmidt, R., Dawkins, S. T. and Rauschenbeutel, A., "Optical interface created by laser-cooled atoms trapped in the evanescent field surrounding an optical nanofiber," Phys. Rev. Lett. 104, 203603 (2010).

[12] Petersen, J., Volz, J. and Rauschenbeutel, A., "Chiral nanophotonic waveguide interface based on spin-orbit interaction of light," Science 346, 67-71 (2014).

[13] Hoffman, J. E., Ravets, S., Grover, J. A., Solano, P., Kordell, P. R., Wong-Campos, J. D., Orozco, L. A. and Rolston, S. L., "Ultrahigh transmission optical nanofibers," AIP Adv. 4, 067124 (2014).

[14] Yu, H. and Li, B., "Wavelength-converted wave-guiding in dye-doped polymer nanofibers," Sci. Rep. 3, 1674 (2013).

[15] Meng, C., Xiao, Y., Wang, P., Zhang, L., Liu, Y. and Tong, L., "Quantum-dot-doped polymer nanofibers for optical sensing," Adv. Mater. 23, 3770-3774 (2011).

[16] Ta, V. D., Chen, R., Ma, L., Ying, Y. J. and Sun, H. D., "Whispering gallery mode microlasers and refractive index sensing based on single polymer fiber," Laser Photon. Rev. 7, 133-139 (2013).

[17] Li, D. and Xia, Y., "Electrospinning of nanofibers: Reinventing the wheel?," Adv. Mater. 16, 1151-1170 (2004).

[18] Reneker, D. H. and Chun, I., "Nanometre diameter fibres of polymer, produced by electrospinning," Nanotechnology 7, 216-223 (1996).

[19]Li, D., Wang, Y. and Xia, Y., "Electrospinning of polymeric and ceramic nanofibers as uniaxially aligned arrays," Nano Lett. 3, 1167-1171 (2003).

[20] Sun, D., Chang, C., Li, S. and Lin, L., "Near-field Electrospinning," Nano Lett. 6, 839-842 (2006).

[21] Camposeo, A., Persano, L. and Pisignano, D., "Light-emitting electrospun nanofibers for nanophotonics and optoelectronics," Macromol. Mater. Engineer. 5, 487-503 (2013).

[22] Gaio, M., Moffa, M., Castro-Lopez, M., Pisignano, D., Camposeo, A. and Sapienza, R., "Modal coupling of single photon emitters within nanofibre waveguides," ACS Nano 10, 6125-6130 (2016). 
[23] Camposeo, A., Greenfeld, I., Tantussi, F., Pagliara, S., Moffa, M., Fuso, F., Allegrini, M., Zussman, E. and Pisignano, D., "Local mechanical properties of electrospun fibers correlate to their internal nanostructure," Nano Lett. 13, 5056 (2013).

[24]Fasano, V., Polini, A., Morello, G., Moffa, M., Camposeo, A. and Pisignano, D., "Bright light emission and waveguiding in conjugated polymer nanofibers electrospun from organic salt added solutions," Macromolecules 46, 5935- 5942 (2013).

[25] Hou, H., Jun, Z., Reuning, A., Schaper, A., Wendorff, J. H. and Greiner, A. "Poly(p-xylylene) nanotubes by coating and removal of ultrathin polymer template fibers," Macromolecules 35, 2429 (2002).

[26] Greenfeld, I., Arinstein, A., Fezzaa, K., Rafailovich, M. H. and Zussman, E., "Polymer dynamics in semidilute solution during electrospinning: a simple model and experimental observations," Phys. Rev. E 84, 041806 (2011).

[27] Greenfeld, I., Fezzaa, K., Rafailovich, M. H. and Zussman, E., "Fast X-ray phase-contrast imaging of electrospinning polymer jets: measurements of radius, velocity, and concentration," Macromolecules 45, 36163626 (2012).

[28] Morello, G., Moffa, M., Girardo, S., Camposeo, A. and Pisignano, D., "Optical gain in the near infrared by light-emitting electrospun fibers," Adv. Funct. Mater. 24, 5225-5231 (2014).

[29] Wiersma, D., "The physics and applications of random lasers," Nat. Photon. 4, 359-367 (2008).

[30] Krämmer, S., Vannahme, C., Smith, C. L., Grossmann, T., Jenne, M., Schierle, S., Jørgensen, L., Chronakis, I. S., Kristensen, A. and Kalt, H., "Random-cavity lasing from electrospun polymer fiber networks," Adv. Mater. 26, 8096-8100 (2014). 